\title{
Xanthogranulomatous Cholecystitis: Our Clinical Experience
}

\author{
Osman Erdogan ${ }^{1,}$, Alper Parlakgumus ${ }^{2}$, Alper Sozutek ${ }^{3}$, Umit Turan ${ }^{4}$, Zeynel Abidin Tas ${ }^{5}$ and Oktay Irkorucu ${ }^{6}$ \\ ${ }^{1} \mathrm{MD}$, Specialist of Surgical Oncology, Department of Surgical Oncology, Adana City Training and Research Hospital, Adana, Turkey \\ 2MD, Associate Professor, Department of Surgical Oncology, Adana City Training and Research Hospital, Adana, Turkey \\ ${ }^{3} \mathrm{MD}$, Associate Professor, Department of Gastroenterological Surgery, Adana City Training and Research Hospital, Adana, Turkey \\ ${ }^{4} \mathrm{MD}$, Specialist of General Surgery, Department of General Surgery, Adana City Training and Research Hospital, Adana, Turkey \\ ${ }^{5} \mathrm{MD}$, Specialist of Pathology, Department of Pathology, Adana City Training and Research Hospital, Adana, Turkey \\ ${ }^{6}$ MSc, MD, FEBS (hon), FACS, University of Sharjah, College of Medicine, Department of Clinical Sciences, Sharjah, UAE
}

* Corresponding author: Osman Erdogan, Department of Surgical Oncology, Adana City Training and Research Hospital, Adana, Turkey. Tel: +903224559090; Email: osman_erdogan85@hotmail.com

Received 2021 August 01; Revised 2021 August 15; Accepted 2021 November 04.

\begin{abstract}
Background: Xanthogranulomatous cholecystitis (XGC) is a rarely encountered chronic inflammatory condition presenting with severely proliferated fibrotic tissue. It usually spreads the neighboring organs, imitates gallbladder cancer, and may lead to difficulty in cholecystectomy.

Objectives: This study aimed to review the clinical and radiological findings, as well as surgical treatment results of XGC.

Methods: This retrospective study analyzed the clinical features of 36 cases with a diagnosis of XGC on pathology. The patients were operated on in our institute between 2012 and 2019.

Results: The rate of XGC in cholecystectomy patients was found to be $0.6 \%(36 / 5999)$ in the hospital where this study was performed over seven years. The patients were aged 33-87 years, and the median age when the patients were diagnosed was 57 years. Moreover, the majority of the patients $(52.8 \% ; n=19)$ were male (male to female ratio: $1 / 1.11$ ). The XGC was not accompanied by gallbladder carcinoma in any of these cases and could not be diagnosed in any of the patients prior to surgery. Radiological imaging performed before surgery demonstrated cholelithiasis, thickening of the gallbladder wall, and suspicious cancer in 29 (80.6\%), 28 (77.8\%), and 2 (5.6\%) patients, respectively. However, none of the cases of XGC had concomitant gallbladder cancer. In total, 9 (25\%) patients underwent open cholecystectomy, and 27 $(75 \%)$ cases were scheduled to have laparoscopic cholecystectomy; however, $6(16,8 \%)$ of these patients were converted to open cholecystectomy.

Conclusion: Laparoscopic cholecystectomy for XGC is possible; however, it is often difficult due to severe inflammation. Patients with XGC have a higher rate of conversion to open surgery and complications, compared to those with other forms of cholecystitis. The XGC may resemble gallbladder cancer based on the diagnostic imaging findings, and intraoperative frozen section analysis is essential to avoid unnecessarily extended surgery.
\end{abstract}

Keywords: Cholecystectomy, Cholecystitis, Gallbladder, Xanthogranulomatous cholecystitis

\section{Background}

Xanthogranulomatous cholecystitis (XGC) is a chronic inflammatory condition rarely occurring and characterized by severely proliferated fibrotic tissue, increased macrophages containing fats, as well as acute and chronic inflammatory cells of the gallbladder (1). It is mainly featured by gallbladder wall thickening and may adhere to adjacent organs, including liver, duodenum, colon, and omentum; in addition, it even creates fistula (1). It is clinically and radiologically difficult to distinguish $\mathrm{XGC}$ from chronic cholecystitis and gallbladder carcinoma (GBC) (2). Furthermore, it is challenging to make a firm diagnosis of this condition before and during surgery. Inability to diagnose it correctly causes extensive surgery involving hepatic resection because of the severe fibrosis and adhesions to contiguous organs $(1,2)$. The XGC is treated with open surgery or laparoscopic cholecystectomy. The latter is the most preferred surgical procedure for benign gallbladder disease at present. However, it often fails in the treatment of XGC and results in prolonged surgery, more complications, and more conversion to open cholecystectomy (1-3).

\section{Objectives}

This study aimed to evaluate the clinical and radiological findings, as well as surgical procedures and outcomes of XGC patients treated in our clinic in the last seven years.

\section{Methods}

This retrospective study reviewed the medical records of 5,999 patients who underwent cholecystectomy at the hospital between January 2012 and December 2019. The XGC was detected in 36 out of 5,999 patients in pathological examinations. Its diagnosis was based on disseminated or localized mural features, including histiocytes, cholesterol, lipid depositions, extremely large multinucleated cells reacting to foreign bodies, phagocytosed lipids and bile pigments, producing xanthomatous cells, and acute or chronic inflammatory cells on histological 
examinations. Data stored in the hospital registry system about clinical and radiological features, perioperative observations, surgical procedures, and postsurgical course were also reviewed in this study. In addition, data about age, gender, clinical picture, concurrent jaundice, pancreatitis, preoperative radiological examinations, perioperative findings, and postsurgical complications were gathered. Patients with inaccessible data, those in whom a diagnosis of XGC could not be confirmed by a pathological examination, and cases aged $<18$ years were excluded from the study. The study protocol was approved by the institutional review board of the hospital (IRB No. 20.05.2020/57/871), and written informed consent for surgery was obtained from all patients.

The obtained data were analyzed in SPSS software (version 24.0) (SPSS Inc. Chicago, IL, USA). The results of the data analysis regarding quantitative and nominal variables were shown in mean \pm SD or median values (range), as well as frequencies, respectively.

\section{Results}

Out of 5,999 cholecystectomy cases during seven years, $36(0.6 \%)$ patients had XGC. On the other hand, none of the patients had GBC accompanying XGC. Clinical features of the patients with XGC are shown in Table 1.

All the patients underwent ultrasound examination. Accordingly, computed tomography (CT), magnetic resonance imaging (MRI) with contrast enhancement, MRCP, and positron emission tomography (PET) were obtained from 21 (58.3\%), 5 (13.9\%), 14 (38.9\%), and 1 patient, respectively. The $\mathrm{XGC}$ was not diagnosed in any of the patients before surgery. The most frequent radiological feature was cholelithiasis $(n=29 ; 80.6 \%)$, and none of the patients had intramural nodules. However, hepatic infiltration $(n=2 ; 5.6 \%)$ pericholecystic infiltration $(n=2 ; 5.6 \%)$, pericholecystic abscess ( $n=2 ; 5.6 \%)$, and gallbladder perforation $(n=1 ; 2.8 \%)$ were observed. Furthermore, $2(5.6 \%)$ patients had gallbladder cancer suspected on preoperative radiological examination, and gallbladder wall thickening of more than $4 \mathrm{~mm}$ was observed in 28 (77.8\%) patients (Figure 1).

Types of surgery performed in 36 patients with $\mathrm{XGC}$ are presented in Table 2. Malignancy was suspected in four cases during surgery. Therefore,

\begin{tabular}{lc}
\hline $\begin{array}{l}\text { Table 1. Clinical characteristics and preoperative imaging findings of } 36 \text { patients with Xanthogranulomatous } \\
\text { cholecystitis }\end{array}$ \\
\hline $\begin{array}{l}\text { Age (years) median (range) } \\
\text { Gender (male: female) }\end{array}$ \\
Male & 57 (range: $33-87)$ \\
Female & \\
Cholelithiasis & $19(52.8 \%)$ \\
GB wall thickening $\geq \mathbf{4}$ mm & $17(47.2 \%)$ \\
Focal & $29(80.6 \%)$ \\
Diffuse & $28(77.8 \%)$ \\
Suspicious malignancy & $6(16.7 \%)$ \\
Intramural nodule & $22(61.1 \%)$ \\
Pericholecystic infiltration & $2(5.6 \%)$ \\
Hepatic invasion & 0 \\
Pericholecystic abscess & $2(5.6 \%)$ \\
Gall bladder perforation & $2(5.6 \%)$ \\
CBD stone & $2(5.6 \%)$ \\
Mirizzi syndrome & $1(2.8 \%)$ \\
\hline
\end{tabular}


Figure 1. Thickening of the gallbladder wall in patients with Xanthogranulomatous cholecystitis. A: Abdominal computed tomography scan of a patient with Xanthogranulomatous cholecystitis. B: Specimen of a patient with Xanthogranulomatous cholecystitis. 
intraoperative frozen examinations were performed, which revealed a benign condition in all four cases. Totally, 27 patients underwent surgery through laparoscopy. The surgery could be completed laparoscopically in only 21 (58.3\%) cases; however, it had to be converted to open surgery in 2 (5.6\%) patients because of severe pericholecystic adhesions.

Choledochoduodenal fistula required a conversion to open surgery in 2 patients. In one case, open cholecystectomy with open common bile duct (CBD) exploration was carried out due to CBD injury. This patient had Strasberg type E1 bile duct injury, circumferential injury to the main bile duct, and leakage. The patient underwent choledochoduodenostomy. In one case, open cholecystectomy with open CBD exploration was carried out due to impacted CBD stone, and open cholecystectomy was carried out in 9 (25\%) patients.

Out of these 9 patients, pericholecystic infiltration $(n=2)$, hepatic invasion $(n=2)$, suspicious malignancy $(\mathrm{n}=2)$, pericholecystic abscess $(\mathrm{n}=2)$, and gallbladder perforation with abscess $(n=1)$ were noted in the cases. Open cholecystectomy with gallbladder bed wedge resection was performed in $2(5.6 \%)$ patients because of the inflammatory mass extending into the liver tissue. Frozen section of the specimen was done in $6(16.7 \%)$ patients during surgery owing to the high probability of malignancy before and after surgery, as well as the presence of a mass. All 6 cases revealed a benign condition. The maximum length of surgery was $240 \mathrm{~min}$ (mean: 104.7 \pm 48.5 ), and the maximum length of hospitalization was 23 days with $5.67 \pm 4.4$ days on average (range: 2-23 days).

Intraoperative and postoperative complications are shown in Table 2. In total, 6 (16.7\%) patients had complications. Of all these patients experiencing postoperative complications, 2 (5.6\%) cases had surgical site infections managed with drainage and antibiotics, and one patient experienced pulmonary embolism treated with medical support therapy. Another patient having postoperative acute pancreatitis was also treated with conservative treatment. The case with Strasberg type E1 bile duct injury was discharged 10 days after the operation without any problems. One patient who developed a postoperative biliary fistula was treated with ERCP and stent. Biliary fistula regressed within two weeks after ERCP. None of the patients in this series died.

\begin{tabular}{lc}
\hline Table 2. Operative outcomes for 36 patients with Xanthogranulomatous cholecystitis \\
\hline Initial laparoscopic approach & $21(58.3 \%)$ \\
Initial open approach & $9(25 \%)$ \\
Open conversion & $6(16.7 \%)$ \\
Intraoperative frozen & $6(16.7 \%)$ \\
Gallbladder bed wedge resection & $2(5.6 \%)$ \\
Operation time (min) & $104.72 \pm 48.5(50-240)$ \\
Hospital stay (days) & $5.67 \pm 4.4(2-23)$ \\
Intraoperative and postoperative complications & $1(2.8 \%)$ \\
CBD injury & $1(2.8 \%)$ \\
Biliary fistula & $2(5.6 \%)$ \\
Wound infections & $1(2.8 \%)$ \\
Acute pancreatitis & $1(2.8 \%)$ \\
Pulmonary emboli & \\
\hline
\end{tabular}

\section{Discussion}

The XGC is an uncommon inflammatory condition that was first described as fibro Xanthogranulomatous cholecystitis by Christensen and Ishak in 1970 (4). It has been stated in the literature that the incidence of all inflammatory conditions related to the gallbladder is $0.7 \%-13.2 \%$. It is a rare inflammatory disorder of the gallbladder presenting with rapidly increasing and damaging inflammation. Different from chronic cholecystitis, diagnosis and surgical treatment of XGC can be difficult since it has aggressive clinical features spreading from the gallbladder wall to the neighboring organs. For this reason, it can be regarded as a clinical condition different from cholecystitis (5). The XGC leads to acute or chronic inflammation in the gallbladder, linked with the introduction of bile pigments and cholesterol into the gallbladder wall. Rupture of Rokitansky-Aschoff sinuses, small mucosal ulcerations, gallstones, and presence of bacteria in the bile eliminated by macrophages may play a role in this serious inflammation. Furthermore, XGC has benign histological features but a severe clinical picture; in addition, it is clinically important due to difficulty in its differentiation from gallbladder cancer (6).

It has been reported in the literature that XGC has an incidence varying between $0.7 \%$ and $11 \%$, and emerges at the ages between 44 and 63 years. The evidence about gender incidence is conflicting, and some authors have demonstrated female predominance, whereas others have shown male predominance (1-5). In the current study, XGC had an incidence of $0.60 \%$ and was found to appear at the age of 58.2 years on average without a difference between genders.

The XGC does not have specific clinical symptoms and resembles acute and chronic cholecystitis. Upon hospital admission, patients with 
XGC often have the symptoms of acute cholecystitis with complications, such as obstructive jaundice, acute biliary pancreatitis, or empyema of the gallbladder. In addition, it may appear as a gallbladder mass imitating GBC.

In the current study, $47.2 \%$ of the cases $(17 / 36$ patients) had an acute presentation and were admitted to the emergency department; moreover, abdominal pain was the most common complaint $(\mathrm{n}=35 ; 97.2 \%)$. In a study conducted by Eriguchi et al. with a large sample, the incidence of acute cholecystitis was found to be $17 \%$ (2). GuzmanValdivia G reported that jaundice was a common symptom (23\%), which was due to Mirizzi syndrome and choledocholithiasis in $70 \%$ and $30 \%$ of the cases, respectively (3). In the present study, 4 $(11.1 \%)$ patients had choledocholithiasis and 2 (5.6\%) cases suffered from Mirizzi syndrome.

Ultrasonography was used to detect an increase in the gallbladder wall thickness (focal or diffuse), gallstones, and intramural hypoechoic nodules. Parra J.A et al. reported that the findings on ultrasound, including increased thickness, hypoechoic nodules and bands in the gallbladder wall, pericholecystic fluid accumulation, and existence of gallstones may suggest XGC (7). It has been revealed that hypoechoic bands, resulting from the existence of foamy histiocytes in the gallbladder wall, can be the typical property of XGC (8). There was no evidence suggestive of hypoechoic intramural nodules in the patients in the present study .

Gallstones were detected in $80.6 \%$ of XGC cases in this study. Similarly, the incidence of gall stones was 75\%-90\% in XGC patients in several studies (3, 9). Significant findings that could be useful to differentiate XGC from GBC are disseminated thickening of the gallbladder wall, intramural hypoattenuated nodules accompanied by an undisrupted mucosal line, and the lack of clear liver damage (9). In the present study, abdominal CT and MRI were utilized in 21 and 5 patients, respectively, to obtain detailed information and decide an appropriate surgical method .

However, the CT and MRI findings were not considered specific or diagnostic for XGC and still suggested GBC. There was disseminated thickness in the gallbladder wall in most of the patients (61.1\%), and three patients had the characteristics of XGC on MRI, such as intramural T2-high signal intensity and continuous enhancing mucosal line with preserved mucosal layering, which helped to differentiate it from GBC $(10,11)$.

Radiological examinations do not sufficiently differentiate between XGC and GBC since they demonstrate features varying from slightly thickened walls to widespread pericholecystic inflammation involving the liver and seldom the neighboring organs. For this reason, it could be challenging to distinguish acute and chronic inflammatory conditions from localized GBC. When the liver and neighboring organs are involved, it can be unlikely to distinguish XGC from malignancy. In the present series, two patients had suspected malignancy on preoperative CT and MRI and were found to have XGC on the last pathological examination

In a study performed by Makino et al., XGC was shown to have enhanced uptake of fluorodeoxyglucose (FDG) on PET scanning imitating GBC. They reported that PET examination is insufficient to differentiate them (12). The XGC shows a positive image due to FDG uptake by active inflammatory cells $(2,12)$. In the present series, FDG PET with a SUVmax (maximum standardized uptake) value suggested GBC in one patient with suspicious malignancy; however, the diagnosis of XGC was confirmed on the last histopathological examination. Among the significant features of XGC imitating GBC are intraoperative macroscopic findings in addition to clinical and radiological ones. The XGC can damage the circumference of the gallbladder, cause severe fibrotic adhesions in adjacent organs, and create fistula with neighboring structures imitating GBC (13). For these reasons, perioperative examination and palpation alone can offer confusing information, and it is crucial to perform a frozen section examination to establish whether XGC is benign and decide on an appropriate surgical method (14).

In the present study, the intraoperative frozen examination was carried out in six cases since preoperative and perioperative findings suggested malignancy. Proving that the disease is not malignant prevents radical surgery and resultant morbidity. However, it should be noted that XGC may imitate GBC or accompany it. It has been noted in the literature that XGC has a stronger relationship with GBC than with gallstone disease and chronic cholecystitis (15). The incidence of co-occurring XGC and GBC ranges from $5 \%$ to $12.5 \%$ (16). Owing to the high possibility of false-negative results in these cases, the diagnosis is established by performing histopathological examinations (17). The inflammation in XGC is assumed to exhibit its effect via the metaplasia-dysplasia-neoplasia chain (18). In addition, focal Xanthogranulomatous alterations have been reported to emerge in the gallbladder wall that displays neoplastic invasion in $30 \%$ of the cases of GBC (18). Nevertheless, several studies have revealed no relationship between XGC and GBC $(13,15)$. There was no GBC coexisting with $\mathrm{XGC}$ in the present series. The treatment of choice for XGC is surgery. The abundance of adhesions to surrounding organs accompanied by marked wall thickening should strongly suggest XGC. These features create two significant challenges for the surgeon. Firstly, GBC must be excluded since it requires invasive surgery. Secondly, surgical dissection may cause complications increasing 
duration and type of surgery, such as open vs. laparoscopic. There have been several studies suggesting that laparoscopy should not be performed for XGC due to the likelihood of cancer, as well as difficulty and higher rates of conversion to open surgery $(1,3,19)$. In the present series, 9 (25\%) patients underwent open cholecystectomy, and laparoscopic cholecystectomy scheduled for 21 $(58.3 \%)$ patients was converted to open cholecystectomy in $6(16.7 \%)$ patients.

The laparoscopic surgery for XGC is associated with a high rate of conversion varying from $10 \%$ to $80 \%(1,3,19-21)$. In this series, higher conversion from laparoscopic cholecystectomy to open surgery $(22.2 \%)$ could be attributed to technical difficulties, including difficult anatomy, adhesions, and hemorrhage which is similar to the results of several earlier studies $(1,3,19-22)$.

The complication rates after surgery for XGC have been reported to be higher than those after standard laparoscopic or open cholecystectomy (1, $3,13,19-22)$. This tendency was also observed in the present study. In fact, the postsurgical complication percentage was $16.7 \%$ (6/36 patients), which was significantly high when compared to those from the usual cholecystectomy series $(1,3,13,19-22)$. It can be ascribed to the technical difficulty of gallbladder removal. In the present series, the high complication rate caused prolonged hospital stays. The maximum duration of hospitalization was 23 days, which was caused by the biliary fistula. Despite comparatively greater morbidity and prolonged hospital stay, none of the patients experiencing complications needed to have reoperation under general anesthesia, and they healed with no mortality.

\section{Conclusion}

In conclusion, $\mathrm{XGC}$ is a rare inflammatory gallbladder disease the diagnosis and treatment of which are challenging. Laparoscopic cholecystectomy for XGC is possible but often difficult due to the presence of severe inflammation. Patients with XGC have a higher rate of conversion to open surgery and complications, compared to those with other forms of cholecystitis. The XGC may resemble gallbladder cancer based on the clinical and diagnostic imaging findings. Intraoperative frozen section analysis is useful for distinguishing between XGC and cancer and is important for avoiding unnecessarily extended surgery.

\section{Acknowledgments}

None.

\section{Footnotes}

Authors' contributions: Osman Erdogan contributed to critical revision, drafting, and writing the article. Alper Parlakgumus contributed to conception and interpretation of data. Alper Sozutek contributed to design and literature research. Umit Turan was responsible for interpretation and critically important intellectual content. Zeynel Abidin Tas contributed to pathological interpretation, design, and literature research. Oktay Irkorucu approved the final version to be submitted.

Ethical Approval: Ethical codes and principles of the Helsinki Declaration have been followed during the study. Moreover, written permission and Ethics Committee permission (IRB No. 20.05.2020/57/871) were obtained from the Adana City Training and Research Hospital.

Funding/Support: The author(s) received no financial support for the research, authorship, and publication of this study from any public or private organization or institute.

Financial Disclosure: None

Conflicts of Interest: The author(s) declare that there is no conflict of interest with respect to the research, authorship, and/or publication of this manuscript.

\section{References}

1. Park JW, Kim KH, Kim SJ, Lee SK. Xanthogranulomatous cholecystitis. Is an initial laparoscopic approach feasible? Surg Endosc. 2017;31(12):5289-5294. doi: 10.1007/s00464-0175604-z.

2. Eriguchi N, Aoyagi S, Tamae T, Kanazawa N, Nagashima J, Nishimura K, et al. Xanthogranulomatous cholecystitis. Kurume Med J 2001;48:219-221. doi: 10.2739/kurumemedj.48.219.

3. Guzman-Valdivia G Xanthogranulomatous cholecystitis in laparoscopic surgery. J Gastrointest Surg 2005;9(4):494-497. doi: 10.1016/j.gassur.2004.09.036.

4. Christensen AH, Ishak KG. Benign tumors and pseudotumors of the gallbladder. Report of 180 cases. Arch Pathol 1970;90:423-432.

5. Güneș Y, Bostancl Ö, İlbar Tartar R, Battal M Xanthogranulomatous Cholecystitis. Is Surgery Difficult? Is Laparoscopic Surgery Recommended? J Laparoendosc Adv Surg Tech A. 2021;31(1):36-40. doi: 10.1089/lap.2020.0334.

6. Neychev V, Ivanova V, Dikov $T$, Todorov G. Diffuse Xanthogranulomatous Cholecystitis. Master of Disguise. Cureus. 2018;17;10(4):e2492. doi: 10.7759/cureus.2492.

7. Parra JA, Acinas O, Bueno J, Güezmes A, Fernández MA, Fariñas MC. Xanthogranulomatous cholecystitis: clinical, sonographic, and CT findings in 26 patients. AJR Am J Roentgenol. 2000;174(4):979-83. doi: 10.2214/ajr.174.4.1740979.

8. Singh VP, Rajesh S, Bihari C, Desai SN, Pargewar SS, Arora A. Xanthogranulomatous cholecystitis. What every radiologist should know. World J Radiol. 2016;28;8(2):183-91. doi: 10.4329/wjr.v8.i2.183.

9. Saritas AG, Gul MO, Teke Z, Ulku A, Rencuzogullari A, Aydin I, et al. Xanthogranulomatous cholecystitis. a rare gallbladder pathology from a single-center perspective. Ann Surg Treat Res. 2020;99(4):230-237. doi: 10.4174/astr.2020.99.4.230.

10. Sureka B, Singh VP, Rajesh SR, Laroia S, Bansal K, Rastogi A, Bihari C, et al. Computed Tomography (CT) and Magnetic Resonance (MR) Findings in Xanthogranulomatous Cholecystitis. Retrospective Analysis of Pathologically Proven 30 Cases - Tertiary Care Experience. Pol J Radiol. 2017;22(82):327-332. doi: 10.12659/PJR.901728.

11. Jung SE, Lee JM, Lee $\mathrm{K}$ et al. Gallbladder wall thickening: MR imaging and pathologic correlation with emphasis on layered pattern. Eur Radiol 2005;15:694-701. doi: 10.1007/s00330- 
004-2539-2

12. Makino I, Yamaguchi $\mathrm{T}$, Sato $\mathrm{N}$, Yasui $\mathrm{T}$, Kita $\mathrm{I}$. Xanthogranulomatous cholecystitis mimicking gallbladder carcinoma with a false positive result on fluorodeoxy glucose PET. World I Gastroenterol 2009;15(29):3691-3693. doi: 10.3748/wjg.15.3691.

13. Srinivas GN, Sinha S, Ryley N, Houghton PW. Perfidious gallbladders-a diagnostic dilemma with xanthogranulomatous cholecystitis. Ann R Coll Surg Eng 2007;89(2):168-172. doi: 10.1308/003588407X155833.

14. Feng L, You Z, Gou J, Liao E, Chen L. Xanthogranulomatous cholecystitis: experience in 100 cases. Ann Transl Med. 2020;8(17):1089. doi: 10.21037/atm-20-5836.

15. Howard TJ, Bennion RS, Thompson JE Jr. Xanthogranulomatous cholecystitis: a chronic inflammatory pseudotumor of the gallbladder. Am Surg 1991;57(12):821-824.

16. Kishore R, Nundy S, Mehrotra S, Metha N, Mangla V, Lalwani S. Strategies for Differentiating Gallbladder Carcinoma from Xanthogranulomatous Cholecystitis-a Tertiary Care Centre Experience. Indian J Surg Oncol. 2017;8(4):554-559. doi: 10.1007/s13193-017-0677-7.

17. Yamagiwa, H. Dysplasia of gallbladder. Its pathological significance. Acta Pathol Jpn 1987;7:747-754

18. Yamaguchi K, Enjoji M. Carcinoma of the gallbladder. A clinicopathology of 103 patients and a newly proposed staging. Cancer 1988;62:1425-1432. doi: 10.1002/10970142(19881001)62:7<1425::aid-cncr2820620730>3.0.co;2-t.

19. Qasaimeh GR, Matalqah I, Bakkar S, Al Omari A, Qasaimeh M. Xanthogranulomatous cholecystitis in the laparoscopic era is still a challenging disease. $J$ Gastrointest Surg. 2015;19(6):1036-42. doi: 10.1007/s11605-015-2818-z

20. Takeda Y, Tomimaru Y, Yokota Y, Noguchi K, Noura S, Imamura $\mathrm{H}$, et al. Outcomes of laparoscopic cholecystectomy for xanthogranulomatous cholecystitis. Mol Clin Oncol. 2019; 11(3):279-284. doi: 10.3892/mco.2019.1884

21. Makimoto S, Takami T, Hatano K, Kataoka N, Yamaguchi $T$, Tomita M, et al. Xanthogranulomatous cholecystitis: a review of 31 patients. Surg Endosc. 2020;27. doi: 10.1007/s00464020-07828-6.

22. Yucel O, Uzun MA, Tilki M, Alkan S, Kilicoglu ZG, Goret CC. Xanthogranulomatous Cholecystitis: Analysis of 108 Patients. Indian J Surg. 2017;79(6):510-514. doi: 10.1007/s12262-0161511-0. 